(C) 1996 IEEE. Personal use of this material is permitted. However, permission to reprint/republish this material

for advertising or promotional purposes or for creating new collective works for resale or redistribution to servers

or lists, or to reuse any copyrighted component of this work in other works must be obtained from the IEEE.

\title{
HALOS OF INTENSE PROTON BEAMS *
}

\author{
Robert D. Ryne, Salman Habib and Thomas P. Wangler \\ Los Alamos National Laboratory \\ Los Alamos, NM 87545 USA
}

\begin{abstract}
Beam halo is an important issue for accelerator driven transmutation technologies and other applications of $\mathrm{CW}$ proton accelerators. These projects include, for example, the accelerator transmutation of waste, accelerator-based conversion of plutonium, accelerator production of tritium, and the development of a next-generation spallation neutron source. To keep radioactivation within acceptable limits these accelerators must operate with a very low beam loss (less than a nanoampere per meter). Beam loss is associated with the presence of a low density halo far from the beam core. Understanding the physics of halo production and determining methods to control beam halo are important to these projects. In recent years significant advances have been made, both analytically and computationally. In the following we describe recent developments in beam halo theory and simulation, including results from multi-million particle simulations.
\end{abstract}

\section{THE BEAM HALO ISSUE}

Beam halo is an important issue for many proposed projects including the accelerator transmutation of waste (ATW), accelerator-based conversion of plutonium (ABC), accelerator production of tritium (APT), and the development of nextgeneration accelerator-driven spallation neutron sources. All of these projects utilize proton linacs with currents of order 100 $\mathrm{mA}$ and energies of order $1 \mathrm{GeV}$. Small beam losses in the linac and in the high energy beam transport section following the linac can produce radioactivation which can degrade accelerator components and hinder or prevent hands-on maintenance. Much beam loss is due to the formation and interception of a low density beam halo at a large radial distance ( 4 or more times the rms beam radius) from the beam core. At $1 \mathrm{GeV}$, losses must be kept below $1 \mathrm{nA} / \mathrm{m}$ (preferably $0.1 \mathrm{nA} / \mathrm{m}$ ) so that hands-on maintenance can be performed shortly after accelerator shutdown. To design accelerators in this ultra-low loss regime we must understand the causes of beam halo.

\section{THE PARTICLE-CORE MODEL IN A CONSTANT FOCUSING CHANNEL}

Early studies of mismatched charged particle beams showed that such beams could undergo emittance growth and develop a large halo surrounding the beam core [1][2]. Beam mismatch is now believed to be a major source of halo formation. A popular model used to study beam halo is the particle-core model of halo evolution[3]-[8]. In this model halo particles interact with a beam core that is assumed to oscillate because of an initial ra-

\footnotetext{
*Work supported by the U.S. Department of Energy, Office of High Energy Physics and Office of Mathematics, Information and Computer Science
}

dial mismatch; the fields inside the core are roughly proportional to $r$ (in the two-dimensional case), while they are inversely proportional to $r$ outside the core. Thus, a halo particle moving in and out of the core sees a strongly time-dependent nonlinear field superposed on a linear external focusing field. As one might imagine such a system exhibits a variety of dynamical phenomena including chaos. In the particle-core model the core is assumed to have a radial density profile that does not change except in an rms sense. In other words, the beam is always a KV distribution, or always a Gaussian, etc., but the rms size of the beam is allowed to change in accord with the rms envelope equations. In addition to this approximate treatment of the core, the model is not self-consistent since the halo particles do not affect the motion of the core. However, as will be shown below results from the particle-core model are in excellent agreement with results from high resolution particle simulations. A useful way to study this model is to make a stroboscopic plot, in which test particles are plotted once each cycle of the core oscillation. (This technique was first used in halo studies by Lagniel [4][5].) As an example, consider the case of a mismatched KV beam. Figure 1 shows a stroboscopic plot of 32 test particles that were initialized with 16 on the $x$-axis and 16 on the $p_{x}$-axis. The main features of the plot are: (1) a central region that has an extent somewhat larger than the core radius; (2) a large amplitude region where particles exhibit betatron motion perturbed by the core space charge; (3) a period-2 resonant region associated with the fixed points to the left and right of the central region; and (4) a separatrix with an inner branch that encloses the central region and outer branches that separate the period- 2 resonance from the betatron-like trajectories. The period-2 resonance is a parametric resonance corresponding to the fact that resonant particles have an oscillation frequency which is one half the envelope frequency, as has been shown analytically by Gluckstern [7]. In Figure 1 the separatrix is actually a narrow chaotic band, and the outer edge of the band has the approximate shape of a peanut. This "peanut diagram" provides a useful picture for describing halo formation: if particles in an initially well-defined core reach the separatrix (by transport mechanisms not included in the model), then they will be carried to large amplitudes along the outer branch of the separatrix. Also, since a real beam would not have an exactly uniform core, the injected beam could already have a low density tail that extends into the resonance region, and under the dynamics of the model these particles would would be carried to large amplitude and form a halo.

Despite its simplicity, the particle-core model in a constant focusing channel predicts a maximum halo amplitude that is in excellent agreement with high resolution particle simulations run on the CM-5 massively parallel computer at the Los Alamos Advanced Computing Laboratory [9]. Figure 2 shows particle simulation results for a mismatched KV beam having the same 


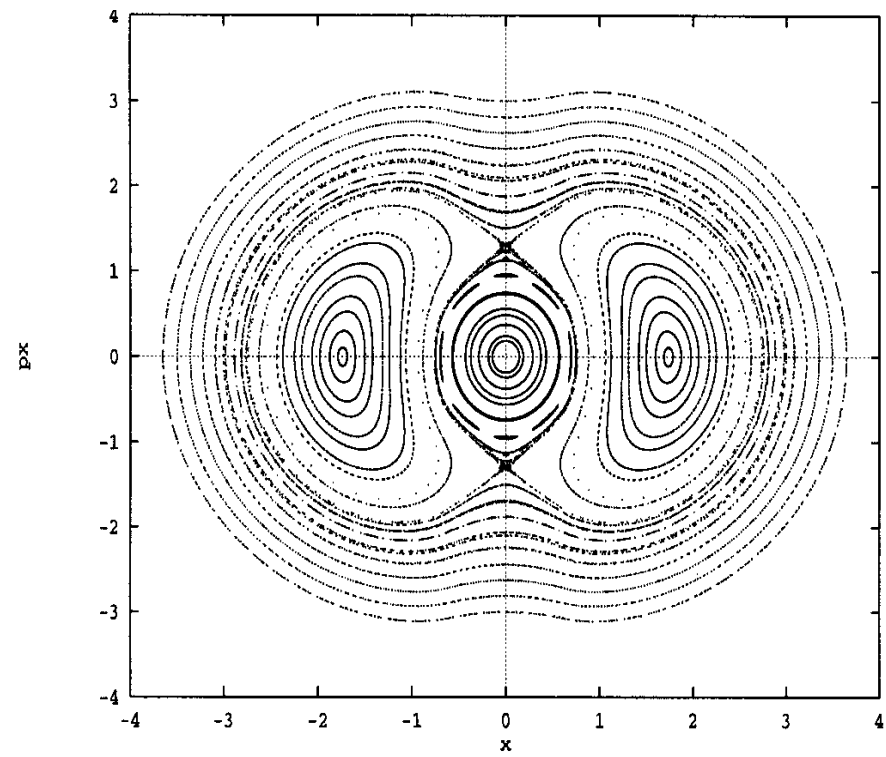

Figure 1. Stroboscopic phase space plot based on the particlecore model with a tune depression of 0.5 and an initial radius 0.62 times the matched radius. The location of 32 test particles is plotted every time the beam envelope reaches a minimum, for a total of 1000 oscillations.

parameters as Figure 1 (a tune depression of 0.5 and an initial beam size that is 0.62 times the matched value). Though the initial distribution has the property that it is uniform in $\left(x, p_{x}\right)$ space, it is unstable for the parameters chosen, and the resulting phase space of Figure 2 is highly nonuniform. (The threshold for this instability has recently been determined analytically by Gluckstern and his colleagues [10].) The first curve bounding the chaotic band in Figure 1 is also shown in Figure 2 for comparison. The CM-5 results show that for this simple configuration (an axially symmetric beam in a constant focusing channel) the maximum particle amplitudes are in excellent agreement with the amplitude of the separatrix in the particle-core model.

\section{THE PARTICLE-CORE MODEL IN A QUADRUPOLE CHANNEL}

The application of the particle-core model to a periodic focusing channel is much more complicated than in a constant focusing channel: In a constant focusing channel there is one frequency driving the dynamics, namely the frequency of the oscillating core; the same is true for a matched beam in a periodic transport system. But a mismatched beam in a periodic channel does not normally oscillate at a single frequency, and this means that stroboscopic plots, which were so useful in illuminating the underlying physics in the constant focusing case (e.g. the period-2 resonance) are not as applicable here. It should be noted, however, that if one linearizes the mismatch then it is possible to excite a single "even" or "odd" mode by a careful choice of initial conditions. This has been done by Lagniel [11].

In the case of a matched beam with little or moderate tune depression, i.e. a tune depression between 1 (no space charge) and 0.5 , the envelope flutter does not appear to be a source of halo formation. Consider for example a matched $\mathrm{KV}$ beam in a

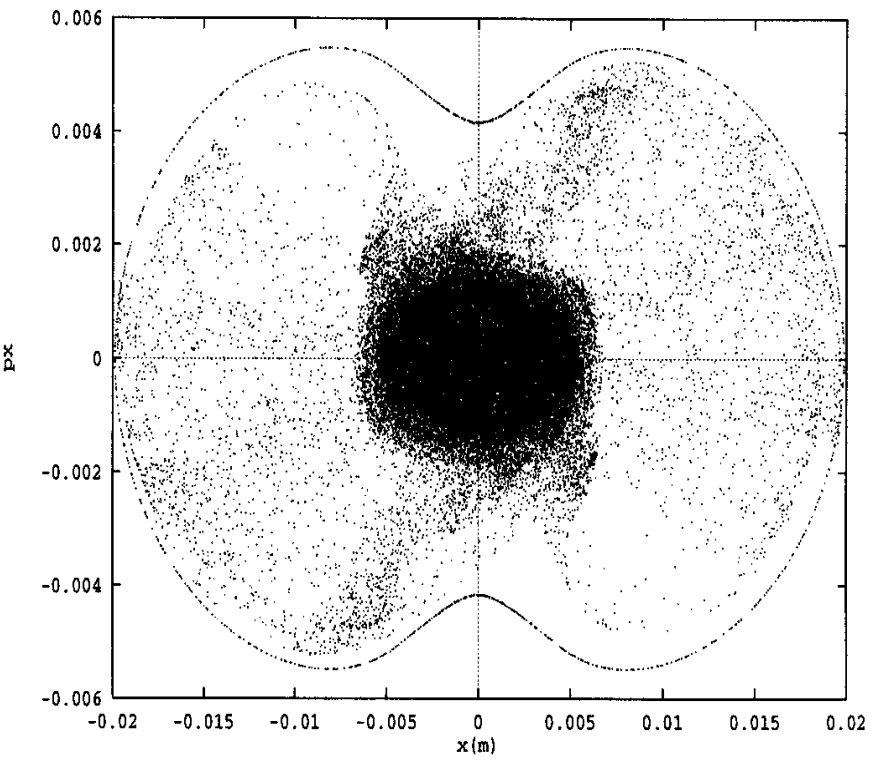

Figure 2. Beam phase space from a 2 million particle simulation on the CM-5 (65536 points are plotted). The outer peanutshaped set of points were obtained from the particle-core model. The CM-5 results show that, for this configuration (an axially symmetric beam in a constant focusing channel), the particlecore model provides a good estimate of the maximum particle amplitudes.

FODO channel with a zero-current phase advance per focusing period of 70 degrees/cell which is depressed by space charge to 35 degrees/cell. A stroboscopic plot for a collection of test particles in such a system is shown in Figure 3; the data points are recorded at the center of each horizontally focusing quadrupole where the matched horizontal beam size is $x_{\text {edge }}=4.6 \mathrm{~mm}$ (i.e. $x_{\mathrm{rms}}=2.3 \mathrm{~mm}$ ). It is clear that the period-2 resonant structure is not present in this case. Though other resonances and weak chaos are present, they do not provide a path by which particles can be transported to large amplitudes. Figure 4 shows particle simulation results for an initially rms matched Gaussian beam in this channel after 22 periods. A large amplitude halo is not present. In contrast, Figure 5 shows the situation when the initial beam has the horizontal and vertical rms envelopes too small by a factor of 0.6 . Now a significant halo is present. It is worth noting that in the matched case the emittance growth (due to charge redistribution) is only $6 \%$, while in the mismatched case it is approximately a factor of 2.

Since most of the projects mentioned previously will operate with somewhat modest space charge (i.e. tune depressions greater than 0.5) envelope flutter in matched beams is not expected to cause halo formation. However, it is important to note that, in the more space charge dominated regime, matched beams in FODO channels can in fact develop halos [12]. This has important ramifications for heavy ion fusion drivers.

\section{NUMERICAL MODELING OF BEAM HALO USING MASSIVELY PARALLEL PROCESSORS}

For many of the projects mentioned above the fraction of particles lost in the accelerator must be kept below one part in $10^{5}$. 


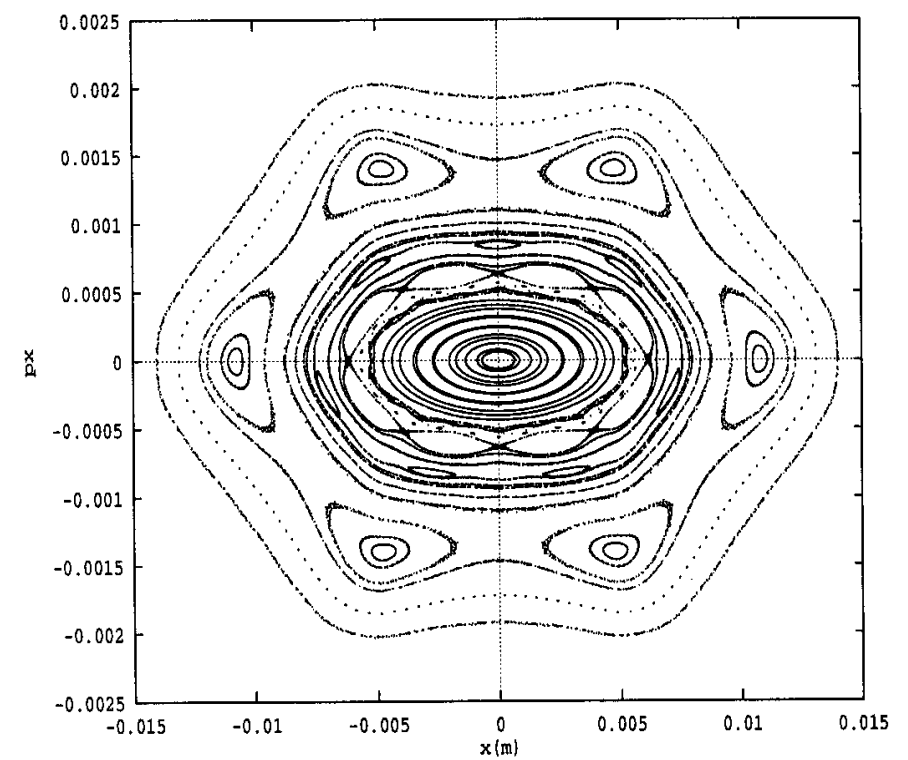

Figure 3. Stroboscopic phase space plot based on the particlecore model for a matched $\mathrm{KV}$ beam in a quadrupole channel. Zero-current phase shift/cell and depressed phase shift/cell are $\sigma_{0}=70 \mathrm{deg}, \sigma=35 \mathrm{deg}$, respectively.

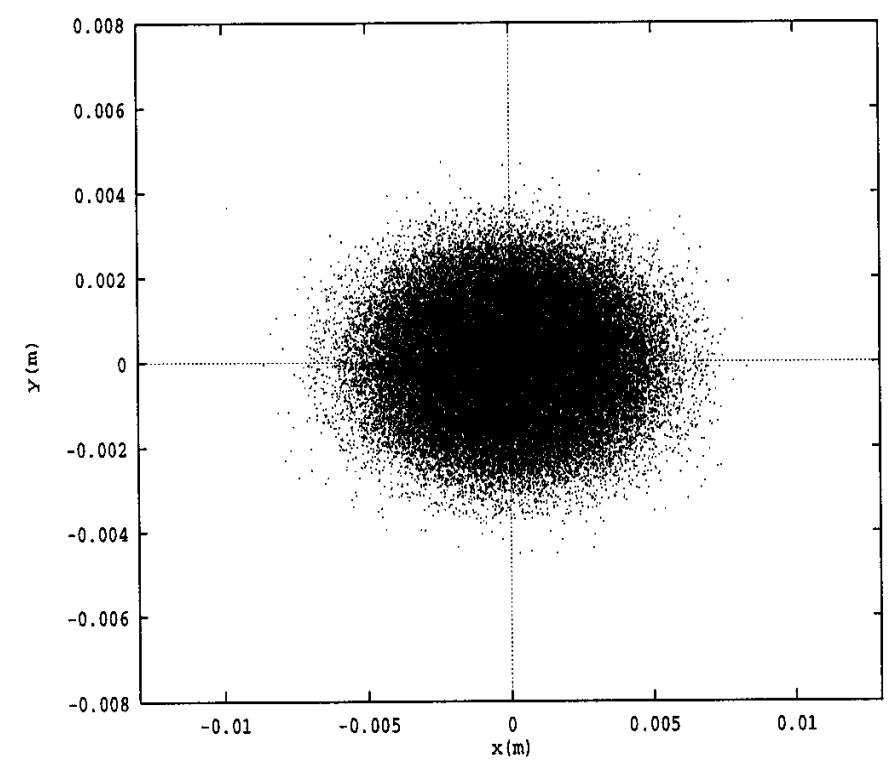

Figure 4. Simulation results showing the beam phase space after 22 focusing periods in a FODO channel. The initial distribution is an rms matched Gaussian beam. ( $\sigma_{0}=70 \mathrm{deg}, \sigma=35 \mathrm{deg}$ )

This suggests that one would like to perform numerical simulations with at least $10^{7}$ particles: If the simulation resulted in there being 100 particles in the halo (corresponding to a fractional loss of $10^{-5}$ ), then the error associated with that number would be roughly $1 / \sqrt{100}=10 \%$, which is adequate for beam halo studies. (Of course, one has to question whether or not the codes contain all the relevant physics needed to accurately predict halo formation.) Such large scale simulations are well suited to massively parallel processors. Particle simulation codes that are used to study beam halo have been developed for the CM-5 at the Los Alamos Advanced Computing Laboratory [13]. The codes perform numerical integration

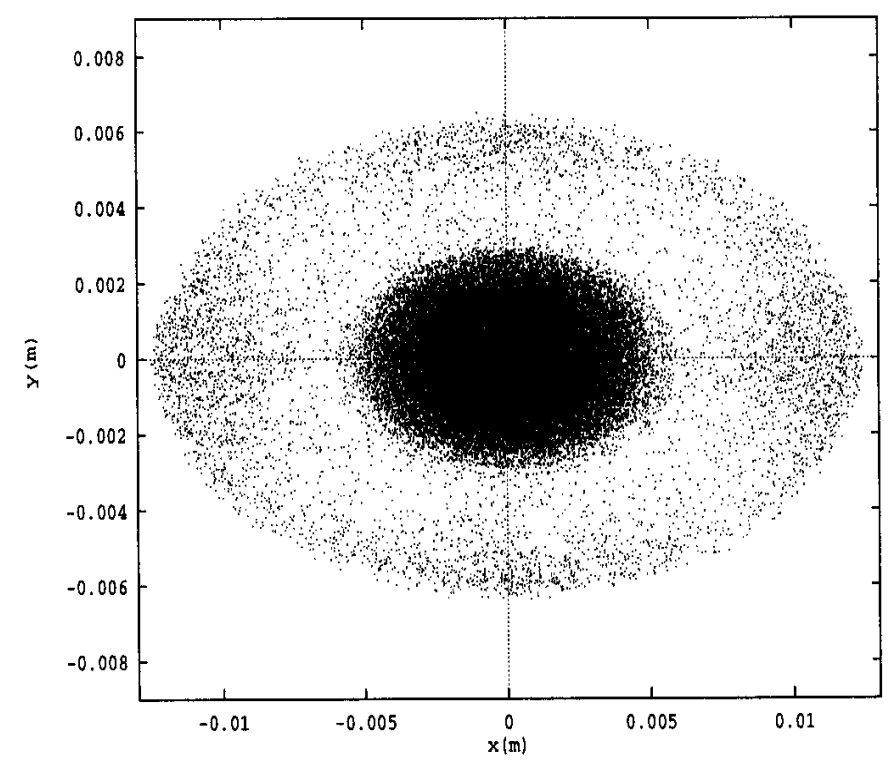

Figure 5. Simulation results showing the beam phase space after 22 focusing periods in a FODO channel. The initial distribution is an rms mismatched Gaussian beam. ( $\sigma_{0}=70 \mathrm{deg}$, $\sigma=35 \mathrm{deg})$

of the particles' equations of motion using a second order or fourth order symplectic integration algorithm [14][15]. In a onedimensional (radially symmetric) version of the code the space charge field is found using Gauss's law, and it involves simply using a mathematical library routine for ordering the particles in radius. In two- and three-dimensional Particle-In-Cell versions of the codes an area weighting scheme is used for the charge deposition and field interpolation; for performance reasons this is implemented using a somewhat complicated scheme that was originally developed for cosmological simulations [16].

Besides using particle simulations, it is possible to solve the Vlasov/Poisson equations directly, and this has also been implemented on the CM-5 in one and two dimensions. A Vlasov/Poisson code solves the equation

$$
\frac{\partial f}{\partial t}+\left(\vec{p} \cdot \partial_{\vec{x}}\right) f-\left(\nabla V \cdot \partial_{\vec{p}}\right) f=0
$$

where $f(\zeta, t)$ is a distribution function on phase space $(\zeta=$ $(\vec{x}, \vec{p}))$. The potential $V$ is a sum of an externally applied potential and a space charge potential which is obtained selfconsistently from Poisson's equation. To solve this equation on the CM-5, we utilize a spectral method coupled with split-operator symplectic integration algorithms [14]. For example, a second-order accurate stepping algorithm for the Vlasov/Poisson equation is given by

$$
f(\zeta, t)=\mathcal{M}(t) f(\zeta, t=0) .
$$

where the mapping $\mathcal{M}$ is given by

$$
\mathcal{M}(t)=e^{-\frac{t}{2}\left(\vec{p} \cdot \partial_{\vec{x}}\right)} e^{t\left(\nabla V \cdot \partial_{\vec{p}}\right)} e^{-\frac{t}{2}\left(\vec{p} \cdot \partial_{\vec{x}}\right)} .
$$

In the context of symplectic integration algorithms, Yoshida showed how to take an algorithm of order $2 n$ and use it to construct an algorithm of order $2 n+2$ [15]. For example, the above 
second-order algorithm can be used to construct a fourth order algorithm,

$$
f(\zeta, t)=\mathcal{M}\left(z_{0} t\right) \mathcal{M}\left(z_{1} t\right) \mathcal{M}\left(z_{0} t\right) f(\zeta, t=0),
$$

where

$$
\begin{aligned}
& z_{0}=\frac{1}{2-2^{1 / 3}}, \\
& z_{1}=\quad-\frac{2^{1 / 3}}{2-2^{1 / 3}} .
\end{aligned}
$$

Output from a two dimensional Vlasov/Poisson code is shown in Figure 6. The initial distribution was a Gaussian in fourdimensional phase space that was mismatched into a quadrupole channel. The simulation utilized a $128^{4}$ grid for a total of 268 million grid points.

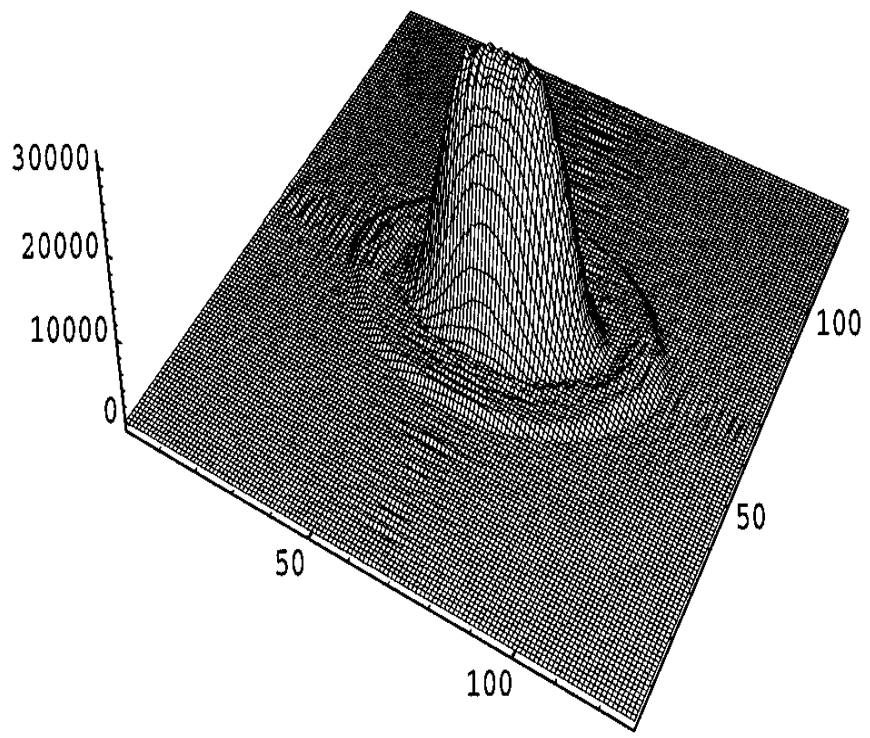

Figure 6. Output from a direct Vlasov/Poisson simulation performed on the CM- 5 showing the beam density at one location in a quadrupole channel. The four-dimensional distribution function was integrated over momenta to obtain the beam density on a $128 \times 128$ grid.

Though this paper emphasizes modeling beam halo, it is worth pointing out that this approach can be applied to several other classical and quantum systems. For example, the Vlasov code can be modified to study gravitating systems. Also, to model the Schrödinger equation,

$$
i \hbar \frac{\partial \psi}{\partial t}=-\frac{\hbar^{2}}{2 m} \nabla^{2} \psi+V \psi,
$$

a second-order algorithm is given by

$$
\psi(\vec{x}, t)=e^{\frac{i t \hbar}{4 m} \nabla^{2}} e^{-\frac{i t}{\hbar} V(\vec{x})} e^{\frac{i t \hbar}{4 m} \nabla^{2}} \psi(\vec{x}, t=0) .
$$

In fact, this is precisely the algorithm used by Feit and Fleck to study a variety of quantum systems, including the vibrational energy levels of triatomic molecules [17]. Using Yoshida's technique, it is in principle possible to obtain high order Schrödinger codes, and a fourth order Schrödinger code has already been developed for the CM-5. Similar codes for evolving the density matrix and the Wigner distribution function, both in the absence and presence of dissipation and noise, have also been developed.

\section{THREE DIMENSIONAL MODELING OF RF LINACS}

Most beam halo studies have previously dealt with one- and two-dimensional systems. In the future it will be important to model beam halo in linacs in three dimensions. High resolution simulations should utilize a few times $10^{7}$ particles and a threedimensional grid (for space charge calculations) whose size is roughly $256^{3}$; this is based on the assumption that, if the beam occupies some reasonable fraction of the grid points (e.g. one half) then there will be a few particles per grid point, as has been found to be adequate for modeling intense beams with PIC codes. (It is interesting to note, however, that for gravitational $\mathrm{N}$-body codes used to model large scale structure formation in the early universe one normally requires only $1 / 8$ particle per grid point.)

Recently there has been much progress in modeling rf linacs, including accurate treatment of the dynamics in the rf gaps [18][19][20]. Also, even when there is acceleration it is still possible to use split operator symplectic integration algorithms to evolve the particles, but it is useful to perform an additional transformation on the Hamiltonian before applying the algorithm. For example, suppose the vector potential for an rf gap is given by

$$
\begin{aligned}
& A_{x}=\frac{e^{\prime}(z)}{2 \omega_{\alpha}} x \sin \left(\omega_{\alpha} t+\theta\right) \\
& A_{y}=\frac{e^{\prime}(z)}{2 \omega_{\alpha}} y \sin \left(\omega_{\alpha} t+\theta\right) \\
& A_{z}=-\frac{1}{\omega_{\alpha}}\left\{e(z)-\frac{r^{2}}{4}\left[e^{\prime \prime}(z)+\frac{\omega_{\alpha}^{2}}{c^{2}} e(z)\right]\right\} \sin \left(\omega_{\alpha} t+\theta\right),
\end{aligned}
$$

where $e(z)$ denotes the spatial part of the electric field at $r=0$,

$$
E_{z}(r=0)=e(z) \cos \left(\omega_{\alpha} t+\theta\right),
$$

and where a prime denotes $d / d z$. Then the transformed Hamiltonian for a system consisting of magnetic quadrupoles and $\mathrm{rf}$ gaps, paraxial in the external fields, is given by $H=H_{x}+H_{y}+$ $H_{\phi}+H_{s c}$, where

$$
\begin{aligned}
H_{x} & =\frac{1}{2 l} \tilde{p}_{x}^{2}+\frac{l \Omega_{x}}{2} \tilde{x}^{2}+\frac{q l g_{m}}{2 p_{o}} \tilde{x}^{2}, \\
H_{y} & =\frac{1}{2 l} \tilde{p}_{y}^{2}+\frac{l \Omega_{y}}{2} \tilde{y}^{2}-\frac{q l g_{m}}{2 p_{o}} \tilde{y}^{2}, \\
H_{\phi} & =\frac{\tilde{p}_{\phi}^{2}}{2 l}+\frac{l \Omega_{\phi}}{2} \tilde{\phi}^{2}, \\
H_{s c} & =\frac{q \Psi}{l \delta \gamma_{o}^{2} \beta_{o} c} .
\end{aligned}
$$

In the above equations $g_{m}$ denotes the magnetic quadrupole gradient, $\Psi$ denotes the space charge potential, $l$ is a scale length, and $p_{o}$ is the momentum of the synchronous particle. The quantities $\Omega_{x}, \Omega_{y}$, and $\Omega_{\phi}$ are given by

$$
\begin{aligned}
\Omega_{x}=\Omega_{y}= & \frac{\left(q / m c^{2}\right)\left(\omega_{\alpha} / c\right)}{2 \beta_{o}^{3} \gamma_{o}^{3}} e \sin \phi_{s} \\
& +\frac{1}{2}\left(1+\frac{\gamma_{o}^{2}}{2}\right)\left(\frac{\left(q / m c^{2}\right) e \cos \phi_{s}}{\beta_{o}^{2} \gamma_{o}^{2}}\right)^{2},
\end{aligned}
$$




$$
\begin{aligned}
& \Omega_{\phi}=\frac{\left(\beta_{o}^{2}+\frac{1}{2}\right)}{\beta_{o}^{3} \gamma_{o}}\left(q / m c^{2}\right)\left(\omega_{\alpha} / c\right) e \sin \phi_{s} \\
& -\frac{3}{2} \frac{q / m c^{2}}{\beta_{o}^{2} \gamma_{o}} e^{\prime} \cos \phi_{s}+\frac{3}{2}\left(1-\frac{\gamma_{o}^{2}}{2}\right)\left(\frac{\left(q / m c^{2}\right) e \cos \phi_{s}}{\beta_{o}^{2} \gamma_{o}^{2}}\right)^{2}
\end{aligned}
$$

where $\beta_{0}$ and $\gamma_{0}$ are the relativistic factors on the synchronous trajectory, and where $\phi_{s}=\omega_{\alpha} t_{o}(z)+\theta$ is the phase of the synchronous particle. The above single particle Hamiltonian was used as a starting point for deriving three-dimensional envelope equations in rf linacs, and this was in turn used to develop a procedure, based on a Hamiltonian formulation of the envelope equations, to find matched beams in rf linacs [21]. The concept of an envelope Hamiltonian has also been used in analytical studies of halo formation [22],[23].

\section{SUMMARY}

Much has been learned about the physics of beam halo, both from a theoretical and computational viewpoint. Most importantly, beam mismatch has been identified as a major source of beam halo. The particle-core model predicts a maximum halo extent which is in excellent agreement with multi-million particle simulations. Also, it has been found that envelope flutter associated with alternating gradient channels is not a significant source of halo formation. Previous work has emphasized oneand two-dimensional analysis and modeling of beam halo. In the future, more difficult three-dimensional issues will have to be addressed.

\section{References}

[1] D. Kehne, M. Reiser, and H. Rudd, 1991 Particle Accelerator Conference, San Francisco, CA, p. 248 (1991).

[2] A. Cucchetti, M. Reiser, and T. Wangler, 1991 Particle Accelerator Conference, San Francisco, CA, p. 251 (1991).

[3] J. O' Connell et al, 1993 Particle Accelerator Confernece, Washington, DC, p. 3657 (May 1993).

[4] J. Lagniel, Nucl. Inst. Meth. A345, 46 (1994).

[5] J. Lagniel, Nucl. Inst. Meth. A345, 405 (1994).

[6] T. Wangler, Los Alamos Report LA-UR-94-1135 (1994).

[7] R. Gluckstern, Phys. Rev. Lett. 73, p. 1247 (1994).

[8] R. Jameson, US-CERN-Japan Topical Course on Accelerator Physics and Technology (1994).

[9] R. Ryne, T. Wangler, and S. Habib, International Conference on Accelerator Driven Transmutation Technologies and Applications, Las Vegas, NV (July 1994).

[10] R. Gluckstern, private communication.

[11] J. Lagniel, private communication.

[12] Q. Qian, R. Davidson and C. Chen, Phys. Plasma, 2, p.2674 (1995).

[13] R. Ryne, and S. Habib, Energy Research Power Users Symposium, Rockville, MD (July 1994).

[14] E. Forest and R. Ruth, Physica D 43, 105 (1990).

[15] H. Yoshida, Phys. Lett. A 150, 262 (1990); E. Forest et al, Phys. Lett. A 158, 99 (1991).

[16] R. Ferrell and E. Bertschinger, Intl. J. Mod. Phys. C (1994).

[17] M. Feit and J. Fleck, Jr., J. Chem. Phys. 78, 301 (1983); M. Feit et al, J. Comp. Phys. 47, 412 (1982); M. Hermann and J. Fleck, Jr., Phys. Rev. A, 38, 38 (1988).
[18] R. Ryne, Los Alamos Report LA-UR-95-1523, April 1995.

[19] J. van Zeijts, these proceedings.

[20] P. Lapostolle, E. Tanke, and S. Valero, Particle Accelerators 44, p. 215 (1994).

[21] R. Ryne, Los Alamos Report LA-UR-95-391, February 1995.

[22] S. Lee and A. Riabko, Phys. Rev. E 51, p. 1609 (1995).

[23] A. Riabko et al., Phys. Rev. E 51, p. 3529 (1995). 